\title{
Different induction of biogenic amine accumulation during cold acclimation in Triticeae genotypes with varying freezing tolerance
}

\author{
Muhammad Ahsan Asghar ${ }^{1,2} \cdot$ Zsuzsa Mednyánszky $^{3} \cdot$ Livia Simon-Sarkadi $^{3} \cdot$ Gábor Kocsy $^{1}$ (i)
}

Received: 15 October 2020 / Revised: 16 December 2020 / Accepted: 18 December 2020 / Published online: 23 January 2021

(c) The Author(s) 2021

\begin{abstract}
The appropriate timing of the accumulation of biogenic amines is very important during cold acclimation due to their direct protective role and their involvement in the signaling processes. The time course of changes in the amount of six of them was compared during a 3-week acclimation period in a freezing tolerant and a sensitive genotype of rye, barley and wheat. In general, a greater and faster cold-induced increase in biogenic amine content was observed in the tolerant genotypes of the three species compared to the sensitive ones. This change was very quick in the case of putrescine, spermidine and cadaverine reaching a maximum after three days in the freezing-tolerant rye genotype. There was a continuous increase in the spermine and tyramine contents during the whole acclimation period in the tolerant wheat genotype while nearly constant levels were detected in the sensitive one. The amount of these two amines exhibited a positive correlation with the level of freezing tolerance in each of the five sampling points. Based on the correlations, a coordinated adjustment of the level of the six studied biogenic amines occurred during the acclimation period which could contribute to the efficient adaptation to cold. In addition, the earlier induction of the biogenic amine accumulation in the freezing tolerant genotypes may contribute to their better cold acclimation.
\end{abstract}

Keywords Barley $\cdot$ Cold-dependent time course $\cdot$ Coordinated changes in amine levels $\cdot$ Rye $\cdot$ Wheat

\section{Introduction}

Biogenic amines play an important role in the control of plant growth and development and their adjustment to stress conditions (Calzadilla et al. 2014; Liu et al. 2015; Chen et al. 2019; Wang et al. 2019). Specifically, biogenic amines are involved in the regulation of cell division, embryogenesis, dormancy termination, regulation of aging, fruit development and stress adaptation. It has been widely documented

Supplementary Information The online version of this article (https://doi.org/10.1007/s40415-020-00690-9).

Gábor Kocsy

kocsy.gabor@atk.hu

1 Agricultural Institute, Centre for Agricultural Research, Martonvásár, Hungary

2 Key Laboratory of Crop Eco-physiology and Farming System in Southwest China, College of Agronomy, Sichuan Agricultural University, Chengdu 611130, China

3 Department of Food Chemistry and Nutrition, Szent István University, Budapest, Hungary that biogenic amine accumulation is an immediate and important response of plants under environmental stressors and through this process plants are able to counteract harmful environmental effects including low-temperature stress (Kovács et al. 2010; Pál et al. 2014).

Cold acclimation induces changes in the content of a large number of metabolites including carbohydrates, amino acids, organic acids and biogenic amines. Biogenic amines, including the monoamine tyramine (Tym), diamines putrescine (Put) and cadaverine (Cad), and the polyamines agmatine (Agm), spermidine (Spd) and spermine (Spm), are low molecular compounds universally present in all living organisms (Calzadilla et al. 2014; Liu et al. 2015; Chen et al. 2019). As polycations, they can directly interact with the negatively charged macromolecules and protect them. Biogenic amines are also regulatory molecules due to their involvement in the signaling and the maintenance of the appropriate redox environment which can be disturbed by the various environmental stresses because of the great accumulation of reactive oxygen species (Wang et al. 2019).

The participation of biogenic amines in the response to low temperature was shown in several plant species (Alcázar 
et al. 2011). The positive effect of biogenic amines on chilling tolerance was demonstrated in tobacco since the endogenous Put and Spd contents positively correlated with the relative growth rate under chilling stress (Wang et al. 2019). In addition, treatment with Put reduced chilling injury in banana (Wang et al. 2003), and foliar application of Put, Spd or Spm increased the freezing tolerance of winter oilseed rape (Jankovska-Bortkevič et al. 2020). A three-week long cold hardening period of wheat at $2{ }^{\circ} \mathrm{C}$ resulted in a large increase in Put and Spd contents (Handa and Mattoo 2010). Similarly, cultivation of various wheat, oat and barley seedlings for $12 \mathrm{~d}$ at $5{ }^{\circ} \mathrm{C}$ induced great accumulation of Put and Spd but not that of Cad and Spm (Pál et al. 2014). These observations indicate the association of biogenic amines with the cold acclimation process.

Although the effect of cold on the level of biogenic amines was investigated earlier, the time course of their accumulation during cold hardening was not compared in various Triticeae genotypes having different level of freezing tolerance. The aim of the present study was to investigate whether the timing of the changes in the amount of biogenic amines exhibits any relationship with the level of freezingtolerance based on the comparison of rye, wheat and barley genotypes with different level of freezing tolerance during a 3 -week hardening period. In addition, we also wanted to find out whether there is a coordinated change in the level of the 6 investigated biogenic amines during hardening.

\section{Materials and methods}

Plant material and cold hardening - One freezing-tolerant and one freezing-sensitive winter rye [Secale cereale $\mathrm{L}$.; LP2 (rye tolerant-RT) and LP6 (rye sensitive-RS)], winter wheat [Triticum aestivum L; Miranovszkaja 808 (wheat tolerant-WT) and GK Tiszatáj (wheat sensitive-WS)] and winter barley [Hordeum vulgare L.; Kold (barley tolerantBT) and Rodnik (barley sensitive-BS)] genotypes were included in our research (Athmer 2012). The seeds of the cereals were germinated for $1 \mathrm{~d}$ at $25^{\circ} \mathrm{C}, 3 \mathrm{~d}$ at $4{ }^{\circ} \mathrm{C}$ and $2 \mathrm{~d}$ again at $25^{\circ} \mathrm{C}$ between wet filter papers. The seedlings were then grown on half-strength modified Hoagland medium for 10 days at $75 \%$ relative humidity and $18 / 15{ }^{\circ} \mathrm{C}$ day/night temperature, with $16 \mathrm{~h}$ illumination at $270 \mu \mathrm{mol} \mathrm{m}^{-2} \mathrm{~s}^{-1}$ photosynthetic photon flux density in growth chambers (Kocsy et al. 2000). The subsequent cold treatment at $2{ }^{\circ} \mathrm{C}$ lasted for 0, 1, 3, 7 or 21 days. Sampling for the biogenic amines' measurement was done in the middle of the photoperiod, in 3 biological replications with three parallels.

Freezing test - After germination the seedlings were transferred to wooden boxes filled with a 2:1:1 (v/v/v) mixture of garden soil, humus and sand and cultivated for $25 \mathrm{~d}$ before successively cold hardened using the program "FDA" (Tischner et al. 1997). The freezing test was carried out as described earlier by the investigation of $5 \times 5$ plants/genotype sown in random block design in each experiment (Sutka 1981). The temperature was gradually decreased $\left(2^{\circ} \mathrm{C} / \mathrm{h}\right)$ before freezing. The rye genotypes were frozen at $-21^{\circ} \mathrm{C}$ or $-19{ }^{\circ} \mathrm{C}$, the wheat genotypes at $-15^{\circ} \mathrm{C}$ or $-12{ }^{\circ} \mathrm{C}$ and the barley genotypes at $-13{ }^{\circ} \mathrm{C}$ or $-10{ }^{\circ} \mathrm{C}$ for $12 \mathrm{~h}$. After freezing, the temperature was gradually increased $\left(2{ }^{\circ} \mathrm{C} / \mathrm{h}\right)$. Then the leaves were cut off and after $14 \mathrm{~d}$ cultivation at $18 / 15^{\circ}$, the re-growth was assessed on a scale between 0 (no re-growth) and 5 (very good re-growth).

Analysis of biogenic amines -The biogenic amine analyses were performed according to a previously published method (Csomos and Simon-Sarkadi 2002). The samples (300 mg fresh weight, $\mathrm{FW}$ ) were extracted with $2 \mathrm{ml}$ cold $10 \%$ trichloroacetic acid (TCA) for $1 \mathrm{~h}$ on a shaker (Laboshake LS 500i, C. Gerhardt GmbH \& Co. KG, Germany). Afterwards, the samples were centrifuged (Heraeus Labofuge 400R, Thermo Fischer Scientific Inc., Germany) for $10 \mathrm{~min}$ at $5000 \mathrm{rpm}$. The supernatants were filtered through a $0.2 \mu \mathrm{m}$ pore membrane filter (Sartorius AG, Germany) and stored at $-20{ }^{\circ} \mathrm{C}$. The determination of different biogenic amines (putrescine-Put, spermidine-Spd, spermineSpm, agmatine —Agm, cadaverine - Cad, thyramine - Tym) was accomplished with an automatic amino acid analyser (Amino Acid Analyzer AAA400, Ingos Ltd., Czech Republic) furnished with an Ostion LG ANB ion exchange column $(6 \times 3.7 \mathrm{~cm})$. They were separated by stepwise gradient elution using a $\mathrm{Na} / \mathrm{K}$-citric buffer system (Ingos Ltd., Czech Republic). At the end, the colorimetric detection was computed after post-column derivatisation with ninhydrin reagent at $570 \mathrm{~nm}$.

Statistical analysis - The statistical analysis was carried out by two-component (treatment, genotypes) analysis of variance (Excel program). Significant differences were determined using $t$-test. The correlations were calculated as described by (Guilford 1950) in order to show relationships between freezing tolerance and the levels of biogenic amines.

\section{Results}

Significant differences were found in freezing tolerance (FT) between the sensitive and tolerant genotypes of each species (Table 1). The largest difference in the freezing-induced damage between the tolerant and sensitive variety was found in wheat (ninefold) and the lowest one in rye (twofold).

The time course of changes in the amount of six biogenic amines (Put, Spd, Spm, Agm, Cad, and Tym) was analysed 
Table 1 The freezing tolerance of the investigated genotypes based on the level of the re-growth of the shoots after the stress

\begin{tabular}{lllll}
\hline Species & Genotype & Sensitivity & Average & SD \\
\hline Rye & LP2 & Tolerant (RT) & $0.54^{\mathrm{b}}$ & 0.16 \\
& LP6 & Sensitive (RS) & $0.24^{\mathrm{a}}$ & 0.06 \\
\multirow{2}{*}{ Wheat } & Miranovszkaja 808 & Tolerant (WT) & $2.86^{\mathrm{c}}$ & 0.96 \\
& GK Tiszatáj & Sensitive (WS) & $0.20^{\mathrm{a}}$ & 0.15 \\
Barley & Kold & Tolerant (BT) & $2.10^{\mathrm{c}}$ & 0.28 \\
& Rodnik & Sensitive (BS) & $0.40^{\mathrm{ab}}$ & 0.15 \\
\hline
\end{tabular}

The re-growth after freezing was assessed on a scale between 0 (no re-growth) and 5 (very good re-growth). The values indicated by different letters are significantly different at $p<0.05$ level (ANOVA, $t$-test, 3 independent experiments, each with 3 parallels)

during a 3-week cold acclimation period. Three of them, Put, Spd and Spm are involved in the same biosynthetic pathways. The Put content exhibited a pronounced, gradual increase in the tolerant variety of all three species which was the greatest and quickest in rye (11.3-fold after $3 \mathrm{~d}$ in RT) (Fig. 1a). This change was smaller and slower in wheat (5.6-fold after $21 \mathrm{~d}$ in WT) and barley (3.5-fold after $21 \mathrm{~d}$ in BT). There was a much smaller increase (maximum twofold) in the sensitive varieties. The Put levels detected after $3 \mathrm{~d}$ and $21 \mathrm{~d}$ of cold acclimation exhibited a very high positive correlations with each other (Table S1). The FT and Put content did not correlate with each other at any sampling point in the studied genotypes. The amount of Spd was greater in most sampling points in the tolerant varieties compared to the sensitive ones (Fig. 1b). Its initial high value did not increase further during cold acclimation except for tolerant rye (1.8-fold increase after $3 \mathrm{~d}$ ) and sensitive barley (2.1-fold increase after $21 \mathrm{~d}$ ) variety. The Spd content even exhibited a great decrease in the tolerant barley variety $(4.5$-fold after $7 \mathrm{~d})$. There was a moderate positive correlation between FT and Spd level ( $r$ : 0.67 ) before the cold treatment (Table S1). The initial concentration of Spm, similarly to Put, was low and gradually increased during cold acclimation in the tolerant varieties of the three genotypes (Fig. 1c). A fast and very large increase was observed in barley (11.4-fold after $3 \mathrm{~d}$ ) and a smaller a slower one in rye and wheat (4.5-fold and 2.7-fold after $21 \mathrm{~d}$ ). In contrast, the amount of Spm was greater only in one sampling point in the sensitive rye (after $3 \mathrm{~d}$ ) and barley (after 21 d) varieties and even decreased in the sensitive wheat variety. The Spm levels detected after $1 \mathrm{~d}$ and $21 \mathrm{~d}$ of cold acclimation had a very high positive correlations with each other (Table S1). The Spm content exhibited a positive correlation with FT throughout the experiment which was moderate after
Fig. 1 The cold-induced accumulation of biogenic amines in Triticeae. $\log _{2}$-values of the amine contents are shown on the heatmaps. The values indicated by asterisks are significantly different from those ones detected at the beginning of the cold acclimation at $p<0.05$ level (ANOVA, $t$-test, 3 independent experiments, each with 3 parallels). RT: tolerant rye, RS: sensitive rye, WT: tolerant wheat, WS: sensitive wheat, BT: tolerant barley, BS: sensitive barley variety
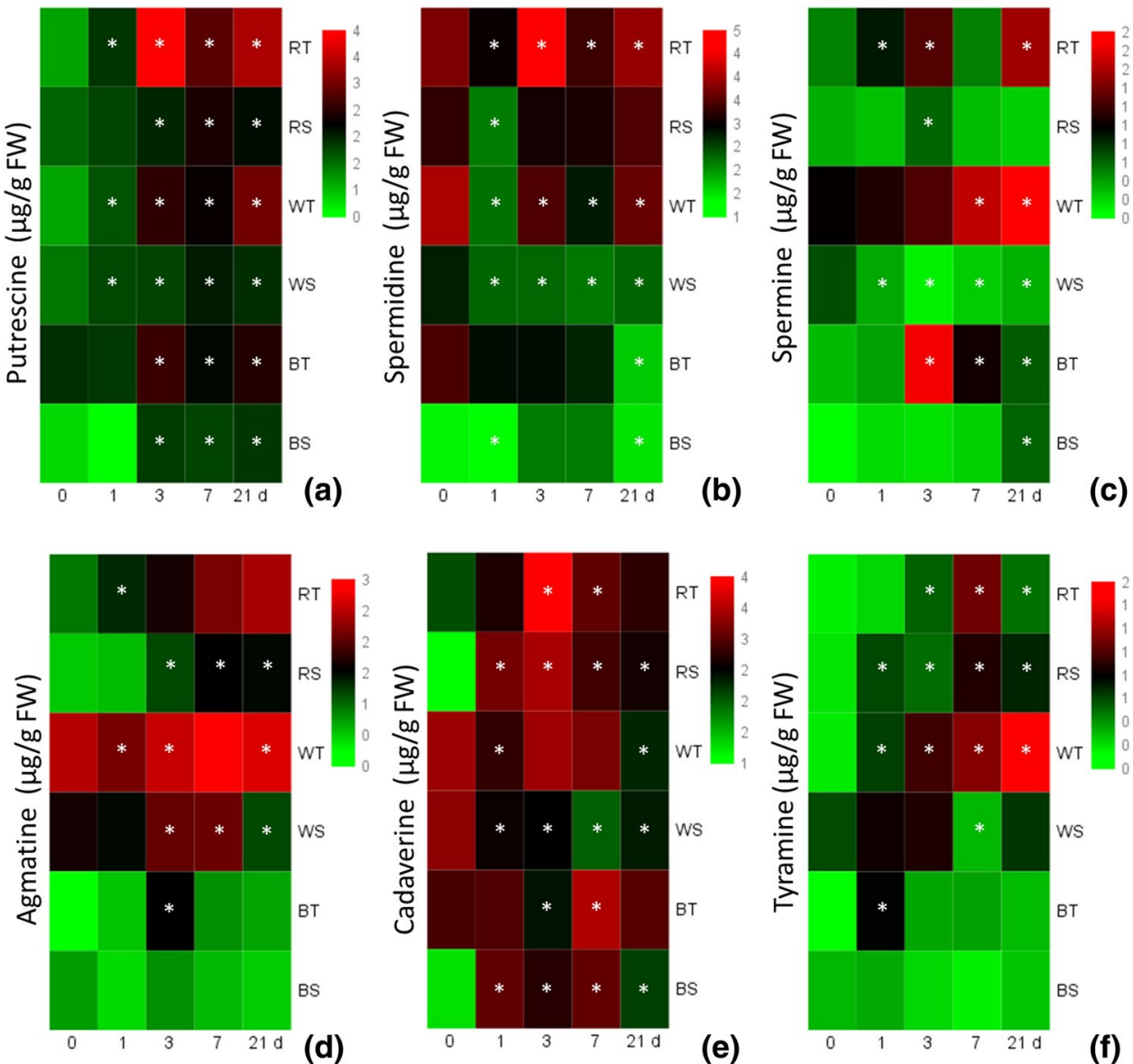

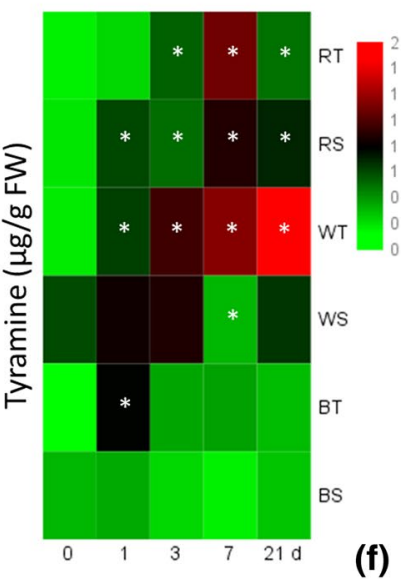


0,1 and $21 \mathrm{~d}$ (r: 0.54, 0.61 and 0.64), high after $3 \mathrm{~d}$ (r: 0.71), and very high after $7 \mathrm{~d}$ (r: 0.97).

The other three investigated polyamines are formed from amino acids by decarboxylation (Agm from Arg, Cad from Lys and Tym from Tyr). The Agm content was greater in the tolerant variety of rye and wheat (Fig. 1d). It increased 4.5-fold in both rye varieties; however, it reached greater level in the tolerant variety compared to the sensitive one. The Agm content was continuously high in wheat and low in barley except for the transient great increase (10.6-fold) after $3 \mathrm{~d}$ in the tolerant barley variety. The Agm contents detected after $0 \mathrm{~d}, 1 \mathrm{~d}, 3 \mathrm{~d}, 7 \mathrm{~d}$ and $21 \mathrm{~d}$ of cold acclimation had a high or very high positive correlations with each other (Table S1). A moderate positive correlation was shown between FT and Agm levels during the whole cold acclimation period. Similarly to Agm, the Cad content increased gradually in rye and reached greater maximum level after $3 \mathrm{~d}$ in the tolerant variety compared to the sensitive one (Fig. 1e). The Cad level was in general high in the other two species with some fluctuations. Its amount was smaller after $21 \mathrm{~d}$ in wheat and greater after $7 \mathrm{~d}$ in barley compared to the starting value. A moderate positive correlation was observed between FT and Cad content after 0 and 7 days ( $r: 0.62$ and 0.67$)$ at low temperature (Table S1.). The cold acclimation induced a greater increase in the Tym content in the tolerant genotypes of all three investigated species varying between eightfold and 13-fold and occurring after 1 $\mathrm{d}$ in barley, after $7 \mathrm{~d}$ in rye and after $21 \mathrm{~d}$ in wheat (Fig. 1f). In the case of sensitive varieties, there was an increase (sixfold, $7 \mathrm{~d}$ ) only in rye. A moderate positive correlation ( $r$ : 0.63 ) between FT and Tym levels was detected after $21 \mathrm{~d}$ cold acclimation (Table S1).

In general, a coordinated adjustment of the biogenic amine levels was observed during cold hardening based on the correlation analysis (Table S2). Although Put is a precursor of Spd and Spm, there was no correlation between the level of Put and the other two biogenic amines except for $1 \mathrm{~d}$ by Spd and $7 \mathrm{~d}$ by Spm. However, the amount of Spm and that of its precursor, Spd exhibited positive correlation during the whole cold acclimation which was high with the exception of the moderate correlation after $3 \mathrm{~d}$. Interestingly, the Tym content exhibited a very high positive correlation $(r \geq 0.9)$ with the Spm content throughout the cold acclimation except for the high correlation after $21 \mathrm{~d}(r$ : 0.77). In addition, a high positive correlation was found between the Tym and Cad levels during the first 3 $\mathrm{d}$ of the cold period and between the Tym and Spd levels after 7 and $21 \mathrm{~d}$. The Cad content showed a high positive correlation with the amount of Spm and Put after 0 and $21 \mathrm{~d}$, respectively, and a high negative correlation with the Agm content after $7 \mathrm{~d}$. There was a very high positive correlation between Agm and Spm levels after 3 and $21 \mathrm{~d}$ cold, and a high one between Agm and Spd levels after $7 \mathrm{~d}$.

\section{Discussion}

In general, a greater and faster accumulation of the examined biogenic amines was observed in the freezing-tolerant varieties compared the sensitive ones in the three investigated Triticeae species. This difference was the most evident in rye except for Tyr. It was usually smaller in the two other species being observed for Put, Spm and Tym in wheat and for Put, Spd and Spm in barley. The earlier adjustment of the amount of the biogenic amines during cold acclimation probably reduces the damages during a subsequent freezing. Thus, its appropriate timing is crucial in the efficient protection against the frost-induced damages. Based on the time course of the changes in the concentrations, Put, Spm, Spd and Cad are probably involved in the initial period of cold acclimation, while Agm and Tym in its later phase. The correlation analysis revealed a coordinated reprogramming of the accumulation of biogenic amines during cold acclimation. Thus, a moderate or high correlation existed between the Spd and Spm levels throughout the experiment, which is not surprising, since Spd is the precursor of Spm. However, no correlation existed between the amounts of Spd and its precursor, Put, and the concentrations of Put and its precursor, Agm (Put is formed through N-carbamoyl-Put from Agm) except for the $1 \mathrm{~d}$ cold treatment. This observation indicates a rapid use of Put and Spd for the synthesis of Spm or their degradation. Interestingly, there was a correlation between the level of Tym and that of three other biogenic amines, Spd, $\mathrm{Spm}$ and Cad, during the whole experiment which further proves the coordinated adjustment of the amounts of the individual biogenic amines. This coordination is important because of the metabolic connections between the individual biogenic amines and their special role in the control of stress response (Singh et al. 2018). It enables a more effective protection against the adverse effects of frost.

The correlation analysis between the cold-induced alterations in biogenic amine levels and FT indicated their involvement in the acclimation process. Their concentration (except for Put) correlated positively with the level of FT at least at one sampling point in the six genotypes of the three Triticeae species. The Spm is the best indicator of this trait since its level showed a positive correlation with FT at all sampling points. Similarly to the present results, the cold-induced accumulation of Put, Spd and Spm was observed in several studies earlier, but only few references are available about the effect of cold on Agm, Cad and Tym levels (Singh et al. 2018). Thus, cold increased Put and Cad contents but not Spd level in wheat (Kovács et al. 2010; Szalai et al. 2009). As observed for the two wheat genotypes in the present study, the concentrations of Agm and Tym also increased in other wheat 
genotypes during cold acclimation (Racz et al. 1996). However, usually only one Triticeae species with one sampling point during cold acclimation was included into the earlier experiments therefore their results cannot show the timing and the general trends of the freezing tolerancedependent changes in the level of the individual biogenic amines during cold acclimation.

In conclusion, the earlier adjustment of the biogenic amine levels in the tolerant varieties of the three Triticeae species contributes to their more efficient protection against frost-induced damages which results in their greater freezing tolerance. This protection is further improved by the coordinated adjustment of the amount of the individual biogenic amines which was shown by the correlation analysis.

Acknowledgements The authors wish to thank Apollónia Horváth and Mónika Fehér for their help in plant cultivation and sampling.

Author contributions GK supervised the experiments. ZM and LSS carried out the measurement of the biogenic amines. MAA made the statistical analysis of the data and prepared the draft of the manuscript. All authors participated in the review and final appovement of the manuscript.

Funding Open Access funding provided by ELKH Centre for Agricultural Research. This research was supported by the National Research, Development and Innovation Office (Grant K 131638). Part of the research was supported by the European Union and co-financed by the European Social Fund (Grant agreement No. EFOP-3.6.3-VEKOP-16-2017-00005).

\section{Compliance with ethical standards}

Conflict of interest The authors declare that they have no conflict of interest.

Open Access This article is licensed under a Creative Commons Attribution 4.0 International License, which permits use, sharing, adaptation, distribution and reproduction in any medium or format, as long as you give appropriate credit to the original author(s) and the source, provide a link to the Creative Commons licence, and indicate if changes were made. The images or other third party material in this article are included in the article's Creative Commons licence, unless indicated otherwise in a credit line to the material. If material is not included in the article's Creative Commons licence and your intended use is not permitted by statutory regulation or exceeds the permitted use, you will need to obtain permission directly from the copyright holder. To view a copy of this licence, visit http://creativecommons.org/licenses/by/4.0/.

\section{Supplementary file1 (XLS70kb) Supplemen- tary file2 (XLSX 24 kb)}

\section{References}

Alcázar R et al (2011) Integration of polyamines in the cold acclimation response. Plant Sci 180:31-38

Athmer B (2012) Differentielle Genexpression in Roggen. Universitätsund Landesbibliothek Sachsen-Anhalt, Weizen und Gerste während der Kälteakklimatisierung und ihr Bezug zur Frosttoleranz

Calzadilla PI, Gazquez A, Maiale SJ, Ruiz OA, Bernardina MA (2014) Polyamines as indicators and modulators of the abiotic stress in plants Plant adaptation to environmental change: Significance of amino acids and their derivatives CABI, Wallingford, UK, pp $109-128$

Chen D, Shao Q, Yin L, Younis A, Zheng B (2019) Polyamine function in plants: metabolism, regulation on development, and roles in abiotic stress responses. Front Plant Sci 9:1945

Csomos E, Simon-Sarkadi L (2002) Characterisation of Tokaj wines based on free amino acids and biogenic amines using ion-exchange chromatography. Chromatographia 56:S185-S188

Guilford J (1950) Creativity Anaer Psychol 5:444-454

Handa AK, Mattoo AK (2010) Differential and functional interactions emphasize the multiple roles of polyamines in plants. Plant Physiol Biochem 48:540-546

Jankovska-Bortkevič E et al (2020) Foliar application of polyamines modulates winter oilseed rape responses to increasing cold. Plants 9:179

Kocsy G, Szalai G, Vágújfalvi A, Stéhli L, Orosz G, Galiba G (2000) Genetic study of glutathione accumulation during cold hardening in wheat-. Planta 210:295-301

Kovács Z, Simon-Sarkadi L, Szúcs A, Kocsy G (2010) Differential effects of cold, osmotic stress and abscisic acid on polyamine accumulation in wheat. Amino Acids 38:623-631

Liu J-H, Wang W, Wu H, Gong X, Moriguchi T (2015) Polyamines function in stress tolerance: from synthesis to regulation. Front Plant Sci 6:827

Pál M et al (2014) Salicylic acid and abiotic stress responses in rice. J Agron Crop Sci 200:1-11

Racz I, Kovacs M, Lasztity D, Veisz O, Szalai G, Paldi E (1996) Effect of short-term and long-term low temperature stress on polyamine biosynthesis in wheat genotypes with varying degrees of frost tolerance. J Plant Physiol 148:368-373

Singh P, Basu S, Kumar G (2018) Polyamines metabolism: A way ahead for abiotic stress tolerance in crop plants. Biochemical physiologi$\mathrm{cal}$ and molecular avenues for combating abiotic stress tolerance in plants. Elsevier, Amsterdam, pp 39-55

Sutka J (1981) Genetic studies of frost resistance in wheat. Theor Appl Genet 59:145-152

Szalai G, Pap M, Janda T (2009) Light-induced frost tolerance differs in winter and spring wheat plants. J Plant Physiol 166:1826-1831

Tischner T, Koszegi B, Veisz O (1997) Climatic programmes used in the Martonvásár phytotron most frequently in recent years. Acta Agron Hung 45:85-104

Wang Y, Lu W-J, Zhang Z-Q, Xie H (2003) ABA and putrescine treatments alleviate chilling injury in banana fruits during storage at $8^{\circ} \mathrm{C}$. J Plant Physiol Mol Biol 29:549-554

Wang Y et al (2019) Polyamines are involved in chilling tolerance in tobacco (Nicotiana tabacum) seedlings. Plant Growth Regul $89: 153-166$

Publisher's Note Springer Nature remains neutral with regard to jurisdictional claims in published maps and institutional affiliations. 